\title{
Analysis of Feedback Parameter with Vibration Sensor on Signal Self-Mixing Interferometry
}

\author{
Novi D. Ariyanti ${ }^{1}$, Endarko ${ }^{1}$, and Agus Rubiyanto ${ }^{1}$
}

\begin{abstract}
In the research aims to analyzed feedback parameter on Self-Mixing Interferometry (SMI) signals. It is because the feedback parameter is one of the determined parameters in SMI vibration sensor research. The experimental apparatus consists of a laser diode as the light source, mirror as a target, collimator as light alignment, and PLX-DAQ as data acquisition executed by Arduino Uno. The principle of data retrieval with the manner of the light source is directed exactly perpendicular to the vibrating target, then reflected and arrested by the detector (photodiode) contained in the laser diode. Light arrested by the photodiode in the form of optical value then automatically converted into voltage value displayed on a personal computer (PC). This feedback on SMI is obtained by variation of the vibration frequency given to a target. The variation of vibration given is $10-100 \mathrm{~Hz}$ with $10 \mathrm{~Hz}$ increment and each frequency is taken 1000 data. Based on the research that has been done then results obtained at the frequency of 10$30 \mathrm{~Hz}$ feedback does not appear because the vibration small so that the vibration sensor is less than optimal. Feedbacks appear at frequencies $40 \mathrm{~Hz}$ and $50 \mathrm{~Hz}$ but are clearly visible at frequencies $50 \mathrm{~Hz}$. While at 60-80 $\mathrm{Hz}$ frequency feedback begins to disappear. The existence of feedback on frequency $50 \mathrm{~Hz}$ indicated that vibration sensors on SMI can be applied to industry and medical field.
\end{abstract}

Keywords-Feedback Parameter, Self-Mixing Interferometry, The Vibration Sensor.

\section{INTRODUCTION}

Self-Mixing Interferometry (SMI) is a method that uses optical technology[1]. The SMI has a very simple interferometry setting. This is because SMI only requires a collimator and does not require optical components like splitter rays[2]. The principle of Self-Mixing Interferometry is a compilation of light from a laser fired towards the target, then several lights reflected by the target and are under arrest detector in the laser. The reflected power and frequency of the target will have a value when compared to initial power and frequency. SMI has an advantage because the structure is simple, easy to align, easy to integrate and noncontact. Based on several advantages, SMI has been widely used in measuring speed, vibration, distance, displacement, biomedicine, 3-dimensional modeling, and various other measurements[3]. The simplest schematic of Self-mixing interferometry with laser diode by Figure 1 below:

\footnotetext{
${ }^{1}$ Novi D. Ariyanti, Endarko, and Agus Rubiyanto are with Department of Physics, Institut Teknologi Sepuluh Nopember, Surabaya, 60111, Indonesia. E-mail: novidwia@gmail.com, endarko@physics.its.ac.id, agus.rubi65@gmail.com.
}

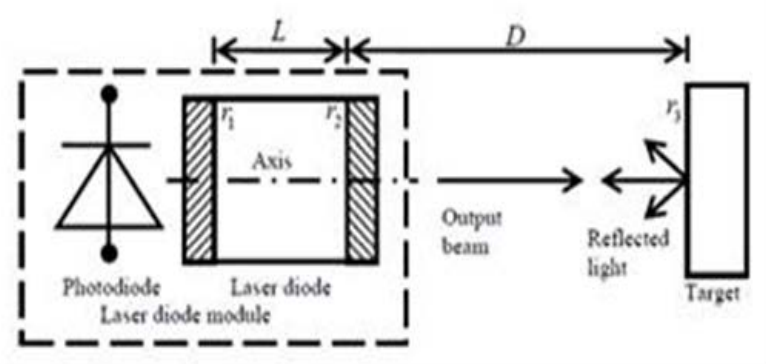

Figure 1. Schematic of Self-mixing interferometry with a laser diode and photodiode[4]

In the SMI scheme, the laser diode is integrated with the photodiode. A high-efficiency laser must be formed from a resonator that has an ability not only to amplify electromagnetic waves but also for feedback. A laser resonator generally consists of two parallel mirrors toward perpendicular to the optical axis. This structure is called the Fabry Perot resonator. Both mirrors have different levels of reflectivity. When the resonator brought into the population inversion state the photons produced by the emission are directly amplified and repeatedly reflected in the two facing faces of the mirrors. This can be seen in Figure 2. At each reflection, the wave transmitted partly through the reflective side. The laser oscillation occurs when the amount of amplification becomes equal to the total amount lost through the resonator side (the mirror face) and the absorption by the crystal[5].

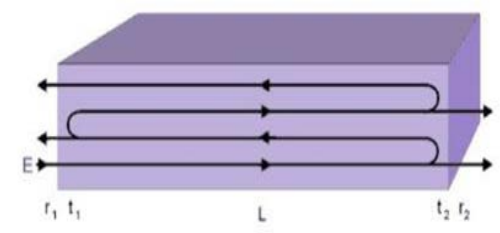

Figure 2. Feedback light in the laser[5]

Feedback parameter (C) is very important because it can distinguish a difference between feedback regimes. The value of the feedback parameter can indicate a particular regime. These regimes are an indicator of the feedback power of optic[6]. Then, in this study, we analyzed the feedback parameters with the vibration sensor in the SMI signal. 


\section{METHOD}

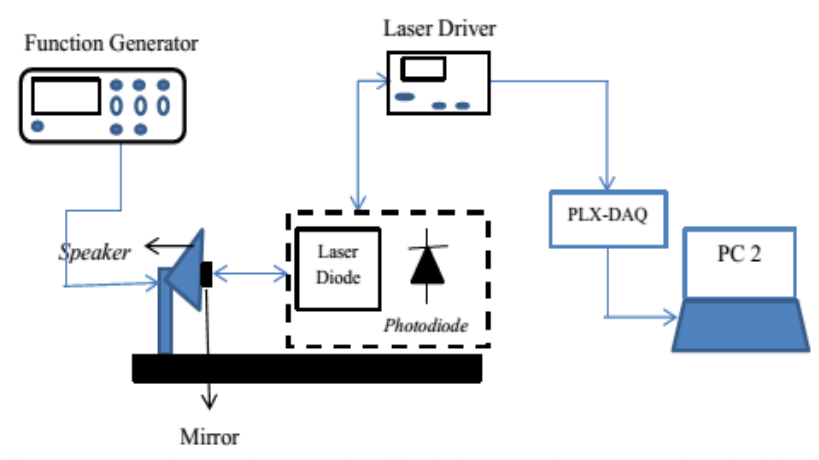

Figure 3. Experimental setup of Self-Mixing Interferometry signal with the vibration sensor

In the experimental setup scheme shown in Figure 3 it consists of Laser diode Thorlabs L785P090 $785 \mathrm{~nm}$ and the photodiode in one component (laser diode as light source and photodiode as a detector), reflective mirror surface attached to the speaker, LDC 210C laser driver as source input current and PC that can display SMI signal. The input current given to the laser driver in this study is $70 \mathrm{~mA}$. The laser light on the vibrating target is reflected and captured by the photodiode. Furthermore, the caught light is transmitted to the laser driver. The target on this system is a mirror inserted on the speaker perpendicular to the laser diode, where they are $1.5 \mathrm{~cm}$ apart and this target as a vibrating object. In the input frequency of $10-80 \mathrm{~Hz}$ of the function, the generator is given to the speakers to cause vibration in the mirror. The output signal is a sinusoidal or cosine signal displayed on a personal computer (PC). Total data were taken for 100 data. So for this measurement is done automatically by Parallax data acquisition (PLXDAQ). So it can be analyzed in the form of a voltage. This is because in the SMI this happens the conversion of optical power into electrical power. After the output voltage is analyzed, then the feedback parameter (C) of the SMI signal can be known

\section{RESULTS AND DISCUSSION}

After the process of measuring SMI-based vibration sensor with a variation of frequency performed. The following is an SMI signal with frequency variation from $10 \mathrm{~Hz}$ to $80 \mathrm{~Hz}$.

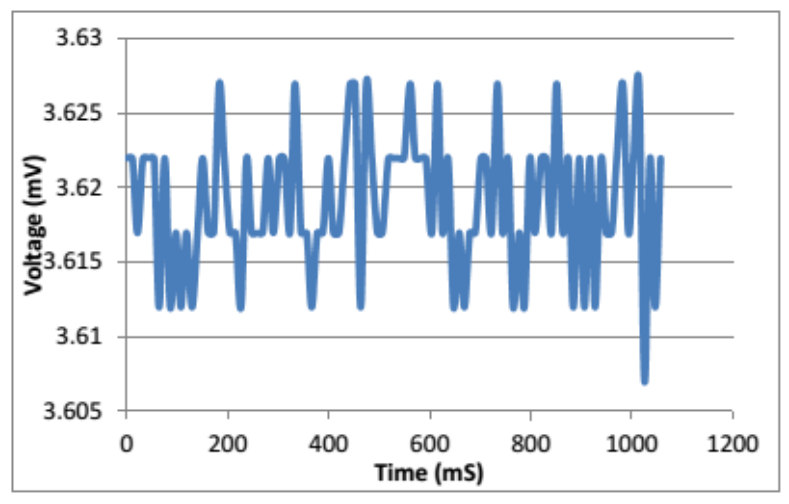

(a)

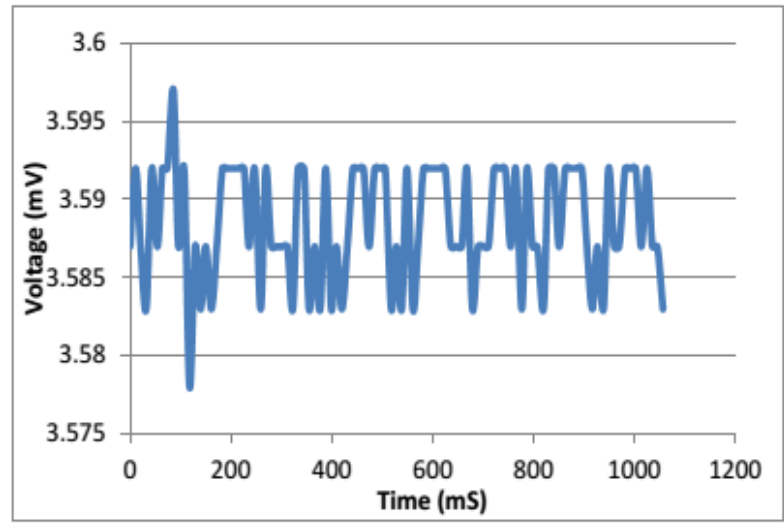

(b)

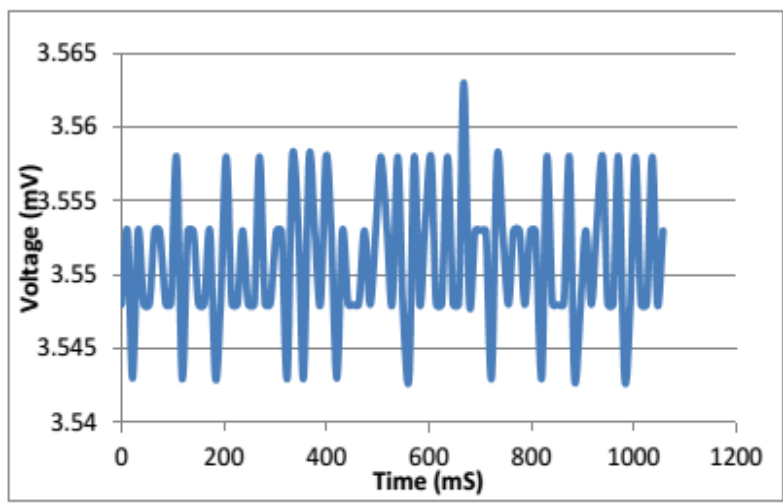

(c)

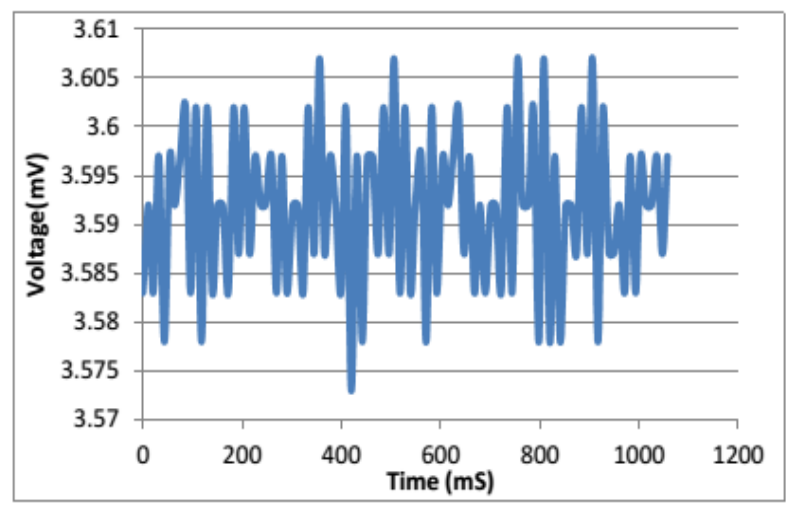

(d)

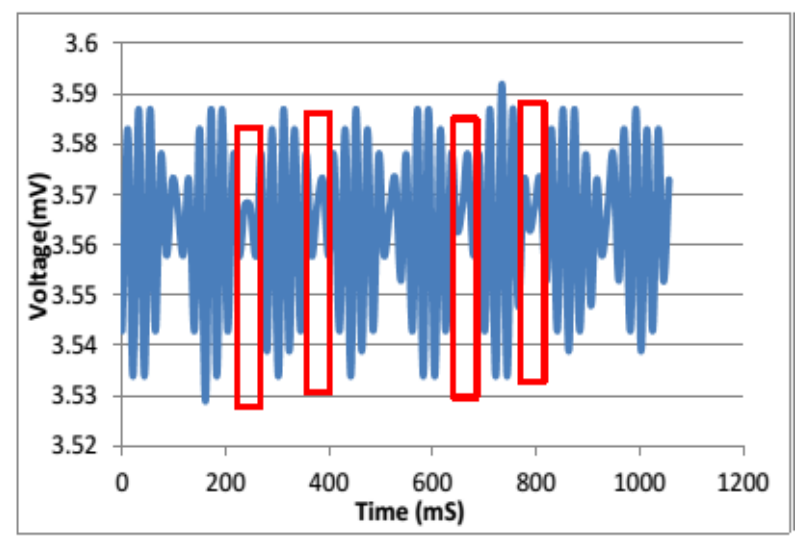

(e) 


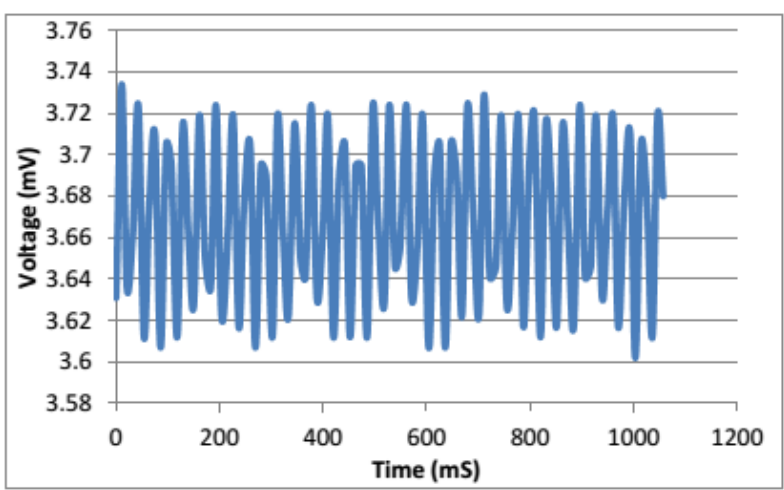

(f)

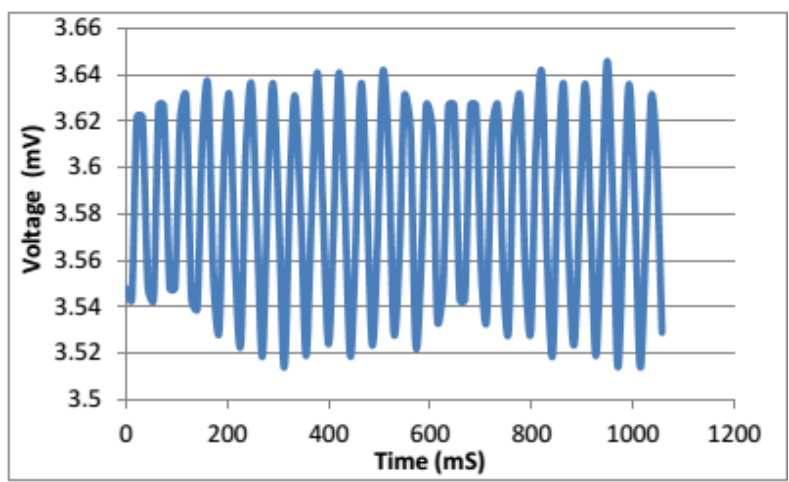

(g)

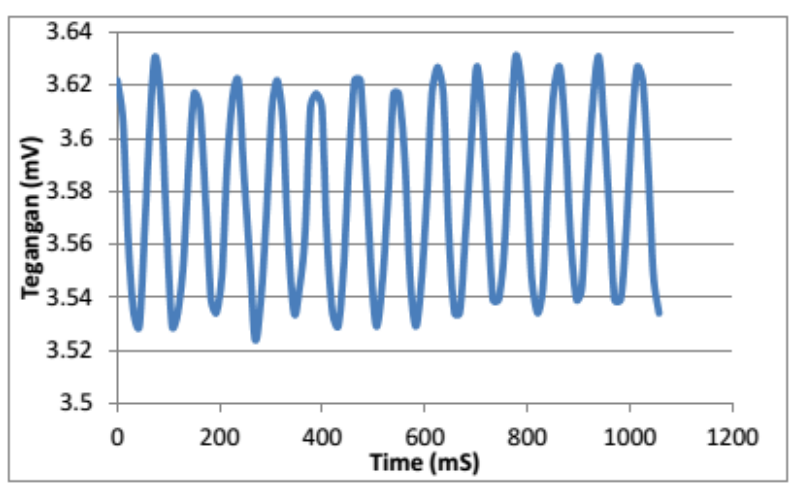

(h)

Figure 4. SMI signals with vibration sensor at frequency range 10-80 Hz. (a) $10 \mathrm{~Hz}$, (b) $20 \mathrm{~Hz}$, (c) $30 \mathrm{~Hz}$, (d) $40 \mathrm{~Hz}$, (e) $50 \mathrm{~Hz}$, (f) $60 \mathrm{~Hz}$, (g) $70 \mathrm{~Hz}$, (h) $80 \mathrm{~Hz}$

In Figure 4, can see the SMI signals with the frequency variation on the vibration sensor. At a frequency of $10 \mathrm{~Hz}$, the SMI signal is irregular while up to $30 \mathrm{~Hz}$ frequency the signal starts to be neat even if feedback does not appear. This is because the vibration is too small so the vibration sensor is less than optimal. Feedback appears at frequency $40 \mathrm{~Hz}$ and $50 \mathrm{~Hz}$ but is clearly visible at $50 \mathrm{~Hz}$. While at $60-80 \mathrm{~Hz}$ frequency feedback began to disappear. The existence of feedback in the signal with the frequency 50 $\mathrm{Hz}$ indicates that the SMI with vibration sensor is in accordance with the characteristics of the SMI signal. According to[6], the feedback parameter (C) is very important because it can distinguish the difference between feedback regimes. The magnitude of the feedback parameter can indicate a particular regime. Where this regime is an indicator of the feedback power (optical feedback).

\section{CONCLUSIONS}

Using a $785 \mathrm{~nm}$ laser diode, the input current in the diode laser is $70 \mathrm{~mA}$ and the frequency variation is $10-80 \mathrm{~Hz}$. The feedback in the SMI signal with a frequency of $50 \mathrm{~Hz}$. This indicates that at that frequency the SMI-based vibration sensor is in accordance with the characteristics of the SMI signal. Then, SMI with vibration sensors can be applied in both industrial and medical fields.

\section{REFERENCES}

[1] W. M. Wang, K. T. V. Grattan, A. W. Palmer, and W. J. O. Boyle, "Self-mixing interference inside a single-mode diode laser for optical sensing applications," J. Light. Technol., vol. 12, no. 9, pp. 1577-1587, 1994.

[2] A. Magnani, D. Melchionni, A. Pesatori, and M. Norgia, "Selfmixing digital closed-loop vibrometer for high accuracy vibration measurements,” Opt. Commun., vol. 365, pp. 133-139, Apr. 2016.

[3] C. Jiang, Z. Zhang, and C. Li, "Vibration measurement based on Multiple Self-Mixing Interferometry,” Opt. Commun., vol. 367, pp. 227-233, May 2016.

[4] L. Martí-López, R. González-Peña, R. A. Martínez-Celorio, and E. E. Ramírez-Miquet, "Vibrometer based on a self-mixing effect interferometer," Rev. Mex. Fis., vol. 58, no. 5, pp. 422-427, 2012.

[5] M. Pospiech and S. Liu, "Technical Introduction to Laser Diodes," LASER DIODE SOURCE, $2004 . \quad$ [Online]. Available: https://www.laserdiodesource.com/laser-diode-technical-overview.

[6] G. Giuliani, M. Norgia, S. Donati, and T. Bosch, "Laser diode selfmixing technique for sensing applications,” J. Opt. A Pure Appl. Opt., vol. 4, no. 6, pp. S283-S294, Nov. 2002. 